\title{
Kac-Moody Monopoles and Periodic Instantons *
}

\author{
H. Garland ${ }^{1}$ and M. K. Murray ${ }^{2}$ \\ ${ }^{1}$ Department of Mathematics, Yale University, Box 2155, Yale Station, New Haven, \\ CT 06520, USA \\ ${ }^{2}$ Department of Mathematics, R. S. Phys. S., The Australian National University, \\ GPO Box 4, Canberra, ACT 2601, Australia
}

\begin{abstract}
It is shown that calorons, or periodic instantons are the same as monopoles with the loop group as their structure group. Their twistor correspondences and spectral data are defined. The spectral data is shown to determine the general caloron.
\end{abstract}

\section{Introduction}

The motivation for this work was the observation, explained in Sect. 1, that the self-duality equations for a periodic instanton could be re-interpreted as the Bogomolny equations for a monopole whose structure group is the loop group if the degree operator ia adjoined to the loop algebra in the usual way. We shall follow the example of Nahm 1983 and call these objects calorons. Except for Sect. 7 we shall concentrate on periodic instantons for $S U(n)$.

Because a caloron has these two interpretations there are two twistor correspondences that can be applied to it. This was first considered for $S U(2)$ by Hitchin in an unpublished work. In Sect. 2 we follow the idea of Hitchin and apply the twistor correspondence for instantons as in Atiyah, Hitchin, and Singer (1978). This shows that the caloron is equivalent to a holomorphic bundle on $\hat{\mathscr{T}}$ the twistor space of $S^{1} \times R^{3}$. If instead the caloron is regarded as a loop group monopole then the twistor correspondence of Hitchin (1982) and Murray (1984) can be applied to show that it is equivalent to an infinite rank holomorphic bundle on the minitwistor space $T P_{1}$. The twistor space $\hat{\mathscr{T}}$ is a $C^{\times}$fibering over $T P_{1}$ and the two holomorphic bundles are related by pushing down these fibres.

A monopole for a compact group has associated to it a collection of algebraic curves indexed by the Dynkin diagram of the group (see Murray, 1984). When two nodes are joined on the Dynkin diagram the intersection of the corresponding curves is split into two pieces. These curves and the splitting are an invariant of the monopole called the spectral data. When the intersections of the curves are finite the monopole is called general and is determined by the spectral data, see Hitchin (1982), Murray (1984), and Hurtubise and Murray (1988b). These spectral curves

* Research supported in part by NSF grant DMS-8506130 
also arise in Nahm's approach to monopoles where the Krichever construction applied to them produces a solution of Nahm's equations from which a monopole is constructed, see Hitchin (1983), Hitchin and Murray (1988), and Hurtubise and Murray (1988a).

The spectral data of a caloron is defined in Sect. 4 and as expected it is a collection of curves indexed by the nodes of the extended Dynkin diagram of the group, that is the Dynkin diagram of the affine Kac-Moody Lie algebra associated to the loop group. In Sect. 6 the spectral data is shown to determine the caloron by an adaptation of an argument in Hurtubise and Murray (1988a).

In Sect. 7 we consider calorons for other loop groups than the loops in $S U(n)$ and also the twisted loop groups. Although no details are given, it can be shown that the spectral data determines the general caloron by adapting the argument in Hurtubise and Murray (1988b).

Unfortunately there is no existence proof for calorons such as Taubes proved for monopoles. Some particular examples are discussed in Chakrabarti (1987). In a later work (Garland, Hurtubise, and Murray) we hope to adapt the results of Hurtubise and Murray (1988a) and Hurtubise (1988) and show that calorons are equivalent to rational maps into the flag manifold of the loop group, and thereby prove their existence.

\section{Calorons}

Let $\Omega L S U(n)$ denote the Lie algebra of all smooth maps from the circle into the Lie algebra of $S U(n)$, equipped with the pointwise bracket. The Lie algebra $\hat{\Omega} L S U(n)$ is then the semi-direct product

$$
\Omega L S U(n) \oplus i R d
$$

with bracket

$$
[X(\theta)+i x d, Y(\theta)+i y d]=[X(\theta), Y(\theta)]-y \frac{\partial X}{\partial \theta}+x \frac{\partial Y}{\partial \theta} .
$$

If $\widetilde{A}(x, t)$ is a connection on $R^{4}$, periodic under $\frac{2 \pi}{\mu_{0}} Z$ acting in the time direction, it can be expanded in an orthonormal basis of one-forms as

$$
\tilde{A}=\Phi(x, t) d t+\sum_{i=1}^{3} A_{i}(x, t) d x^{i} .
$$

If we identify $R / \frac{2 \pi}{\mu_{0}} Z \simeq S^{1}=R / 2 \pi Z$ by $t+\frac{2 \pi}{\mu_{0}} \mapsto t \mu_{0}+2 \pi Z$, then $d \theta=\mu_{0} d t$ and $\frac{\partial}{\partial \theta}$ $=\frac{1}{\mu_{0}} \frac{\partial}{\partial t}$. We can now regard $\Phi$ and the $A_{i}$ as maps from $R^{3}$ into $\Omega L S U(n)$, and if $\tilde{A}$ satisfies the self duality equations, then $A=\sum_{i=1}^{3} A_{i} d x^{i}$ and $\Phi$ satisfy

$$
* F_{A}=d_{A} \Phi-\mu_{0} \frac{\partial A}{\partial \theta},
$$


where $F_{A}$ is the curvature of $A$ considered as an $\Omega L S U(n)$ valued connection form and $*$ is the Hodge star operator on $R^{3}$.

The Bogomolny equations for a connection $\hat{A}=A(\theta)+a d$ and Higgs field $\hat{\Phi}=\Phi(\theta)+\phi d$ taking values in $\hat{\Omega} L S U(n)$ are $* F_{\hat{A}}=d_{\hat{A}} \hat{\Phi}$, and using (1.2) this reduces to the two equations

$$
\begin{gathered}
* F_{A}+i a \frac{\partial A}{\partial \theta} \wedge A=d_{A} \Phi+i \phi \frac{\partial A}{\partial \theta}, \\
* d a=d \phi .
\end{gathered}
$$

If we impose finite action boundary conditions then (after a gauge transformation) the solution of (1.6) is $i a=0$ and $i \phi$ a constant. If the constant is $-\mu_{0}$, then Eq. 1.5 becomes the same as Eq. 1.4. Notice that $\left(0, i \mu_{0}\right)$ is a solution of the $U(1)$ Bogomolny equations which Hitchin (1982) showed defined a line bundle $L^{\mu_{0}}$ on minitwistor space $T P^{1}$.

These calculations show that periodic instantons can be regarded also as Bogomolny monopoles with structure group the semi-direct product $\hat{\Omega} S U(n)$ $=\Omega S U(n) \times U(1)$, where $\Omega S U(n)$ is the loop group or group of smooth maps of the circle into $S U(n)$.

The boundary conditions are, as in the case of finite groups, summarized by saying that the connection and Higgs field extend to the sphere at infinity and there satisfy

$$
d_{A \infty} \Phi^{\infty}+\mu_{0} \frac{\partial A^{\infty}}{\partial \theta}=0
$$

If we trivialize the bundle on the sphere at infinity to make $\Phi^{\infty}$ a map $\Phi^{\infty}: S_{\infty}^{2}$ $\rightarrow L \Omega S U(n)$, then this equation becomes

$$
d \Phi^{\infty}=-[A, \Phi]-\mu_{0} \frac{\partial A}{\partial \theta} .
$$

But the right-hand side of this is the infinitesimal twisted action of $\hat{\Omega} S U(n)$ on $\operatorname{LSSU}(n)$. Hence the image of $\Phi^{\infty}$ is tangent to the orbits of $\hat{\Omega} S U(n)$ so must actually lie in an orbit. If we repeat the Bogomolny trick for Kac-Moody monopoles we have

$$
\begin{aligned}
\operatorname{Action}(A, \Phi) & =\int_{S^{1}} \int_{R^{3}}\left\|* F_{A} \pm\left(d_{A} \Phi+\mu_{0} \frac{\partial A}{\partial \theta}\right)\right\|^{2} d \theta d^{3} x \\
& \mp 2\left\{\int_{S^{1}} \int_{R^{3}}\left(\operatorname{tr} F_{A} \wedge d_{A} \Phi+\mu_{0} F_{A} \wedge \frac{\partial A}{\partial \theta}\right) d \theta d^{3} x\right\} .
\end{aligned}
$$

The second term is topological in nature and by manipulating with the Bianchi identity and applying Stokes theorem it becomes

$$
\int_{S^{1}} \int_{S_{\infty}^{2}} \operatorname{tr}\left(F_{A} \wedge \Phi+\frac{1}{2} \mu_{0} A \wedge \frac{\partial A}{\partial \theta}\right) d \theta .
$$

To understand the meaning of this second term recall that the central extension $\widetilde{\Omega S U}(n)$ has the Lie algebra

$$
\widetilde{\Omega} L S U(n)=\widehat{\Omega} L S U(n) \oplus i R c
$$


with bracket

$$
[X+i x c, Y+i y c]=\left([X, Y], i\left(\int \operatorname{tr} X \frac{\partial Y}{\partial \theta} d \theta\right) c\right) .
$$

To lift the monopole $(A, \Phi)$ to the central extension would be to find an $(a, \phi)$ such that

$$
d a+\operatorname{tr} \int \frac{\partial A}{\partial \theta} \wedge A d \theta=* d \phi+\operatorname{tr} \int \frac{\partial * A}{\partial \theta} \Phi d \theta
$$

(See Sect. 5 for an argument why this should be possible.)

The Killing form for $\widetilde{\Omega} L S U(n)$ is

$$
\left\langle X+x i c+x^{\prime} i d, Y+y i c+y^{\prime} i d\right\rangle=\int_{S^{1}}\langle X, Y\rangle d \theta+x y^{\prime}+x^{\prime} y,
$$

and it has $c$ and $d$ as null vectors and $\langle c, d\rangle=1$.

Asymptotically let

$$
\begin{gathered}
\Phi \sim i\left(\begin{array}{ccc}
\mu_{1} & & 0 \\
0 & \ddots & \mu_{n}
\end{array}\right)+\mu_{0} i d, \\
F \sim \frac{i}{2}\left(\begin{array}{ccc}
k_{1} & & 0 \\
0 & \ddots & k_{n}
\end{array}\right)+\frac{1}{2} k_{0} c .
\end{gathered}
$$

Then if $\hat{F}=F+f i c$ we obtain

$$
f=d a+\operatorname{tr} \int \frac{\partial A}{\partial \theta} \wedge A d \theta
$$

and

$$
\int_{S_{\infty}^{2}} \int_{S^{1}} \frac{\partial A^{\infty}}{\partial \theta} \wedge A^{\infty} d \theta=4 \pi k_{0}
$$

So the topological term becomes

$$
4 \pi^{2} \sum k_{i} \mu_{i}+2 \pi \mu_{0} k_{0}=4 \pi\langle\hat{F}, \hat{\Phi}\rangle .
$$

This calculation shows that, just as for the compact groups, the solutions of the Bogomolny equations minimize the action.

The Kac-Moody group $\widetilde{\Omega} S U(n)$ behaves in many respects like a compact Lie group and has a similar representation theory, a complexification, Borel subgroups, flag varieties, cell decompositions etc. We shall see that these play an important role just as in the case of finite dimensional monopoles (Murray, 1984). First, however, let us consider the twistor correspondence for calorons obtained by regarding them as periodic instantons.

\section{Periodic Instantons}

Using the methods of Atiyah, Hitchin, and Singer (1978) we can construct a twistor space $\hat{\mathscr{T}}$ for $S^{1} \times R^{3}$ and define the usual correspondence between instantons on $S^{1} \times R^{3}$ and holomorphic bundles on $\hat{\mathscr{T}}$. 
The simplest way of constructing $\hat{\mathscr{T}}$ is to recall that the twistor space for $R^{4}$ is $C P_{3}-C P_{1}$ and that there is the fibration

$$
C P_{3}-C P_{1} \rightarrow R^{4} \text {. }
$$

Now $R$ acts on $R^{4}$ by time translation and this action lifts and complexifies to an action of $C$ on $C P_{3}-C P_{1}$. If $\mu_{0} \in R$ then factoring both sides of the fibering (2.1) by the subgroup $\frac{2 \pi}{\mu_{0}} Z$ of $R$ gives the fibering

$$
\hat{\mathscr{T}} \rightarrow S^{1} \times R^{3} .
$$

The quotient of $C P_{3}-C P_{1}$ by $C$ is minitwistor space $T P_{1}$ so $C P_{3}-C P_{1}$ is a $C$ principal bundle over $T P_{1}$. If we quotient by $\frac{2 \pi}{\mu_{0}} Z$ we obtain a $C^{\times} \simeq C /\left(\frac{2 \pi}{\mu_{0}} Z\right)$
bundle over $T P_{1}$ which is $\hat{\mathscr{T}}$.

If $\zeta$ is a co-ordinate for $P_{1}-\{0\}$ and $\frac{1}{\zeta}$ a co-ordinate for $P_{1}-\{\infty\}$, then $X \in T_{\zeta} P_{1}$ can be given co-ordinates $(\eta, \zeta)$, where $X=\eta \frac{\partial}{\partial \zeta}=-\frac{\eta}{\zeta^{2}} \frac{\partial}{\partial\left(\frac{1}{\zeta}\right)}$. So $T P_{1}$ has two coordinate patches $(\eta, \zeta)$ and $\left(-\left(\frac{\eta}{\zeta^{2}}\right), \frac{1}{\zeta}\right)$. Over these patches we can define sections of $C P_{3}-C P_{1} \rightarrow T P_{1}$ by

$$
\varphi_{0}(\eta, \zeta)=[0, i \eta, 1, \zeta], \quad \varphi_{1}(\eta, \zeta)=\left[\frac{-i \eta}{\zeta^{2}}, 0, \frac{1}{\zeta}, 1\right]
$$

where $\left[z_{0}, z_{1}, z_{2}, z_{3}\right]$ is the line in $C^{4}$ through $\left(z_{0}, z_{1}, z_{2}, z_{3}\right)$.

The group $C$ acts on $C P_{3}-C P_{1}$ by $\lambda\left[z_{0}, z_{1}, z_{2}, z_{3}\right]=\left[z_{0}+\lambda z_{2}, z_{1}+\lambda z_{3}, z_{2}, z_{3}\right]$, and so on the overlap of the co-ordinate charts

$$
\varphi_{0}(\eta, \zeta)=[0, i \eta, 1, \zeta]=i \frac{\eta}{\zeta}\left[-\left(i \frac{\eta}{\zeta}\right), 0,1, \zeta\right]=i\left(\frac{\eta}{\zeta}\right) \varphi_{1}(\eta, \zeta)
$$

The transition function of $\hat{\mathscr{T}} \rightarrow T P_{1}$ is obtained by composing with $C \rightarrow C^{\times}, z \mapsto \exp \left(-i \mu_{0} z\right)$, so it must be $\exp \left(\frac{\mu_{0} \eta}{\zeta}\right)$ which is the transition function of the bundle $L^{\mu_{0}}$ introduced in Hitchin (1982).

Any $C^{\times}$bundle such as $\hat{\mathscr{T}}$ has a natural compactification where each fibre has a zero and infinity added to it. One way to see this is to let $C^{\times}$act on $P_{1}$ by $\lambda\left[z_{0}, z_{1}\right]$ $=\left[\lambda z_{0}, z_{1}\right]$ so it fixes $0=[1,0]$ and $\infty=[0,1]$. Then the associated fibration $\mathscr{T}=\left(\hat{\mathscr{T}} \times P^{1}\right) / C^{\times}$defines a $P_{1}$ bundle over $T P_{1}$ which contains $\hat{\mathscr{T}}$ by the mapping which sends $z \in \hat{\mathscr{T}}$ to the orbit under $C^{\times}$of $(z,[1,1])$. This $P_{1}$ bundle has two distinguished sections $\mathscr{T}^{0}$ and $\mathscr{T}^{\infty}$ defined by the fixed points $0, \infty$ of the $C^{\times}$ action on $P_{1}$. Note also that if we realize $\hat{\mathscr{T}}$ as $L^{\mu_{0}}-\{0\}$, where $\{0\}$ is the zero section then $\mathscr{T}$ is the projective bundle $P\left(L^{\mu_{0}} \oplus C\right)$.

The twistor correspondence tells us that a periodic instanton $A$ defines a holomorphic bundle $\hat{E}$ over $\hat{\mathscr{T}}$. Following Hitchin's results for the $S U(2)$ case we shall show in the next section that under appropriate boundary conditions $\hat{E}$ extends to all of $\mathscr{T}$ and has naturally defined filtrations over $\mathscr{T}^{0}$ and $\mathscr{T}^{\infty}$. 


\section{Boundary Conditions}

Let $\tilde{A}$ be a periodic instanton and $(A, \Phi)$ the induced Kac-Moody monopole. Then the boundary conditions we require it to satisfy are firstly that $(A(\theta), F(\theta))$ satisfy the Bogomolny-Prasad-Sommerfield boundary conditions (Murray, 1984) uniformly in $\theta$ and secondly that if $\gamma$ is a line in $R^{3}$, then on the cylinder $\gamma \times S^{1}$ we have for large enough $t$

$$
\nabla_{t}+i \mu_{0} \nabla_{\theta}=\frac{\partial}{\partial t}-i \mu_{0} \frac{\partial}{\partial \theta}+\left(\begin{array}{ccc}
\mu_{1}+\frac{k_{1}}{t} & \ldots & 0 \\
\vdots & \ddots & \vdots \\
0 & \ldots & \mu_{n}+\frac{k_{n}}{t}
\end{array}\right)+C(t, \theta),
$$

where $|C(t, \theta)|<\frac{1}{t^{2}}$ for $t$ a linear parameter on the line. Here $\nabla_{t}$ and $\nabla_{\theta}$ are the covariant derivatives in the direction of the line and the circle. We shall also place some constraints on the values of $\mu_{0}, \mu_{1}, \ldots, \mu_{n}$, and to motivate these let us review some facts about Kac-Moody algebras (see for instance Macdonald, 1981). The algebra $\widetilde{\Omega} L S U(n)$ is defined to be

$$
\widetilde{\Omega} L S U(n)=\Omega L S U(n) \oplus i R d \oplus i R c
$$

with bracket

$$
\left[X+i \lambda d+i \mu c, X^{\prime}+i \lambda^{\prime} d+i \mu^{\prime} c\right]=\left[X, X^{\prime}\right]+\lambda \frac{\partial X^{\prime}}{\partial \theta}-\lambda^{\prime} \frac{\partial X}{\partial \theta}+i\left(\int \operatorname{tr} X \frac{\partial X^{\prime}}{\partial \theta} d \theta\right) c
$$

A Cartan subalgebra is given by

$$
h=L T \oplus i R d \oplus i R c,
$$

where $L T$ denotes the set of constant loops with diagonal entries. Linear forms on $h$ are defined by extending linear forms on $L T$ to be zero on $R d$ and $R c$ and $\delta$ and $\gamma$ defined by

$$
\delta(i d)=1=\gamma(i c), \quad \delta(c)=0=\gamma(d) .
$$

Simple roots for this Cartan subalgebra can be chosen as

$$
\begin{gathered}
\alpha_{0}=\delta-x_{1}+x_{n}, \\
\alpha_{1}=x_{1}-x_{2}, \\
\vdots \\
\alpha_{n-1}=x_{n-1}-x_{n},
\end{gathered}
$$

where $x_{j}\left(\operatorname{diag}\left(i \mu_{1}, \ldots, i \mu_{n}\right)\right)=\mu_{j}$ for $j=1, \ldots, n$.

In the case of finite dimensional monopoles we required that the Higgs field at infinity is in the positive Weyl chamber, that is the simple roots applied to it are greater than zero. Motivated by this we require for Kac-Moody monopoles that

$$
\mu_{0}-\left(\mu_{1}-\mu_{n}\right), \mu_{1}-\mu_{2}, \ldots, \mu_{n-1}-\mu_{n}>0 \text {. }
$$

It follows that $\mu_{0}>\mu_{i}-\mu_{j}$ for any $i \leqq j$, a fact we shall use later. 
We shall assert below that $\left(\nabla_{t}-i \mu_{0} \nabla_{\theta}\right) x(t, \theta)=0$ on any cylinder has (for large enough $t$ ) solutions $x_{i}(t, \theta)$ such that asymptotically for $i=1, \ldots, n$,

$$
\left|x_{i}(t, \theta)\right| \sim \exp \left(-\mu_{i} t\right) t^{k_{j}}, \quad t \rightarrow \infty .
$$

Moreover if locally $C(t, \theta)$ depends on a parameter $w \in T P_{1}$, the solutions $x_{i}$ depend smoothly on $w$ and their derivative with respect to $w$ decays as rapidly as they do.

Why are these facts useful? Recall from the construction of instanton bundles that $\hat{E}$ is just the trivial bundle on $\hat{\mathscr{T}}$ with a $\bar{\partial}$ operator defined by pulling back the connection $A$. The fibre of $\hat{\mathscr{T}} \rightarrow T P_{1}$ over $\gamma$ can be identified with the cylinder in $R^{3}$ $\times S^{1}$ over the line $\gamma$ in $R^{3}$ and the $\bar{\partial}$ operator is just $\nabla_{t}-i \mu_{0} \nabla_{\theta}$. The solutions $x_{i}$ therefore define holomorphic frames $\left\{x_{i}(t, \theta) \mid i=1, \ldots, n\right\}$ in a neighbourhood of infinity which vary smoothly as the fibre varies. If $w$ is a parameter on $T P_{1}$ then applying the $\bar{\partial}$ operator in $w$ to $x_{i}(t, \theta, w)$ we obtain

$$
\sum_{j=1, \ldots, n} B_{j}(t, \theta, w) x_{j}(t, \theta, w),
$$

where $B_{j}(t, \theta, w)$ is a holomorphic function because the $\bar{\partial}$ operator is integrable. Because the derivatives of $x_{i}(t, \theta, w)$ decay as fast as $x_{i}(t, \theta, w)$, it follows that $B_{j}(t, \theta, w) x_{j}(t, \theta, w)$ decays like $\exp \left(-\mu_{i} t\right) t^{-k_{j}}$ and that $B_{j}(t, \theta, w)$ decays like $\exp \left(-\left(\mu_{i}-\mu_{j}\right) t\right) t^{-k_{i}+k_{j}}$ for each $j=1, \ldots, n$.

From (3.7) $\mu_{0}>\mu_{i}-\mu_{j}$ for any, $i \leqq j$. Hence $B_{j}(t, \theta, w)$ decays strictly faster than $\exp \left(\mu_{0} t\right)$. But $B_{j}(t, \theta, w)$ is holomorphic on $\left(C P_{3}-C P_{1}\right) / \frac{2 \pi}{\mu_{0}} Z=\hat{\mathscr{T}}$ in a neighbourhood of infinity, so admits a Laurent expansion as

$$
\sum_{Q \leqq p \leqq Q^{\prime}} d_{p}(w) \exp \left(p\left(\mu_{0} t-i \theta\right)\right) .
$$

It follows that $B_{j}(t, \theta, w)$ must extend across infinity.

If we do this for each $i=1, \ldots, n$ we obtain a matrix of functions $B_{i j}(t, \theta, w)$, and similar considerations show that at $t=\infty$ it is upper triangular. Moreover if we consider different frames on overlapping sets of fibres the induced transition matrix is also upper triangular. This means that when the bundle is extended across $\mathscr{T}^{\infty}$ it has a filtration.

To summarize the bundle $\hat{E}$ extends holomorphically across $\mathscr{T}^{\infty}$. Its restriction $E^{\infty}$ to $\mathscr{T}^{\infty}$ has a filtration

$$
0=E_{0}^{\infty} \subset E_{1}^{\infty} \ldots E_{n}^{\infty}=E^{\infty} .
$$

A similar argument shows that $\hat{E}$ extends across $\mathscr{T}^{0}$, and the bundle $\hat{E}$ extended to all of $\mathscr{T}$ we shall denote by $E$. The restriction $E^{0}$ of $E$ to $\mathscr{T}^{0}$, has a filtration

$$
0=\mathrm{E}_{0}^{0} \subset \mathrm{E}_{1}^{0} \ldots \mathrm{E}_{n}^{0}=E^{0} .
$$

The same kind of arguments used in Hitchin (1982) show that the quotients of these filtrations are

$$
E_{1}^{\infty}=L^{\mu_{1}}\left(-k_{1}\right), \ldots, E_{n}^{\infty} / E_{n-1}^{\infty}=L^{\mu_{n}}\left(-k_{n}\right)
$$

and

$$
E_{1}^{0}=L^{\mu_{n}}\left(k_{n}\right), \ldots, E_{n}^{0} / E_{n-1}^{0}=L^{\mu_{1}}\left(k_{1}\right) .
$$


The real structure on $C P_{3}-C P_{1}$ factors to give a real structure $\sigma: \mathscr{T} \rightarrow \mathscr{T}$ which interchanges $\mathscr{T}^{0}$ and $\mathscr{T}^{\infty}$. This lifts to the bundle $E$ to give

$$
\sigma: E \rightarrow E^{*},
$$

and $\sigma\left(E_{i}\right)=\left(E_{n-i}\right)^{\perp}$, where if $W$ is a subspace of a vector space $V$ then $W^{\perp}$ $=\left\{v^{*} \in V^{*} \mid v^{*}(w)=0\right\}$. It remains now to assert the following

Lemma 3.16. The partial differential equation

$$
\left[\frac{\partial}{\partial t}-i \mu_{0} \frac{\partial}{\partial \theta}+\left(\begin{array}{ccc}
\mu_{1}+\frac{k_{1}}{t} & \ldots & 0 \\
\vdots & \ddots & \vdots \\
0 & \ldots & \mu_{n}+\frac{k_{n}}{t}
\end{array}\right)+C(t, \theta)\right] x(t, \theta)=0
$$
defined on $S^{1} \times\left[t_{0}, \theta\right)$ with $|C(t)|<\frac{1}{t^{2}}$ as $t \rightarrow \infty$ has solutions $x_{j}(t, \theta)$ on some $S^{1}$
$\times\left[t_{1}, \infty\right)$ with

$$
\lim _{t \rightarrow \infty} \sup _{\theta \in S^{1}}\left|\exp \left(-\mu_{i} t\right) t^{-k_{j}} e_{j}-x_{j}(t, \theta)\right|=0,
$$

where $e_{j}$ is the basis vector of $C^{n}$ with a 1 in the $j^{\text {th }}$ entry and zeroes elsewhere. Moreover if $C(t)$ depends smoothly on some parameter $\gamma$ with the bound $|C(t)|<\frac{1}{t^{2}}$ maintained uniformly, then the solutions $x_{j}$ depend smoothly on $\gamma$ and their derivatives with respect to $\gamma$ decay at least as fast as they do.

The proof of this proceeds as for the case without a $\theta$ parameter by letting the functions take their values in Sobolev spaces on the circle. The operator $i \frac{\partial}{\partial \theta}$ is then absorbed into the definition of the Higgs field. The same sort of estimates that yield a proof in the finite dimensional case (Coppel, 1965) work here also.

\section{The Spectral Curves of the Caloron}

The proof of the previous lemma shows that as in Hitchin (1982) outside of a compact set in $T P^{1}$ solutions of (3.17) that look like $e^{\lambda_{j} t}$ as $t \rightarrow \infty$ look like $e^{\lambda_{j} t}$ as $t \rightarrow-\infty$. This means in particular that the bundle $E$ restricted to the fibres of $\pi: \mathscr{T}$ $\rightarrow T P^{1}$ is generically trivial and is non-trivial on fibres above a compact analytic curve in $T P_{1}$. Because the curve is compact it must in fact be algebraic and therefore defined by a section of some $\mathcal{O}(p)$ for $p>0$. Let us denote this curve by $S_{0}$ and calculate the number $p$ by intersecting $S_{0}$ with a generic line in $T P_{1}$.

Let $\mathcal{O}(p, q)$ denote the line bundle on $\mathscr{T}$ with chern class $p$ in the fibre direction and $q$ in the base and $E(p, q)$ the tensor product of this with the vector bundle $E$. Then if $L$ is a fibre we have the exact sequence of sheaves

$$
0 \rightarrow E(-1,-1) \rightarrow E(-1,0) \rightarrow E(-1,0)_{L} \rightarrow 0,
$$

and taking cohomology

$$
\begin{aligned}
0 \rightarrow H^{0}(L, E(-1,0)) & \rightarrow H^{1}(Q, E(-1,-1)) \stackrel{\alpha(L)}{\longrightarrow} H^{1}(Q, E(-1,-1)) \\
& \rightarrow H^{1}(L, E(-1,0)),
\end{aligned}
$$


where $Q$ in $\mathscr{T}$ is the pre-image of the generic line $P_{1}$ in $T P_{1}$. If $L$ is not a jumping line $\alpha(L)$ is an isomorphism and the lines where $E$ jumps are given by the equation $\operatorname{det} \alpha(L)=0$. Hence the number of lines is $h^{1}(Q, E(-1,0))$ the dimension of $H^{1}(Q, E(-1,0))$. From the Riemann-Roch theorem (Hartshorne, 1977)

$$
h^{0}(Q, E(-1,0))-h^{1}(Q, E(-1,0))+h^{2}(Q, E(-1,0))=-c_{2}(E),
$$

but $h^{0}(Q, E(-1,0))=h^{2}(Q, E(-1,0))=0$ so $S_{0}$ is defined by a section of $\mathcal{O}\left(c_{2}(E)\right)$. Moreover if $L$ is a jumping line so is $\sigma(L)$ so $c_{2}(E)$ is even and letting $k_{0}=\frac{1}{2} c_{2}(E)$ we see that $S_{0}$ is defined by a section of $\mathcal{O}\left(2 k_{0}\right)$.

Now we shall assume that when $E$ jumps it splits as $\mathcal{O}(-1) \oplus \mathcal{O}(1) \oplus \mathcal{O} \oplus \ldots \oplus \mathcal{O}$. In the next section we shall show that this is a reasonable condition to expect analogous to those imposed in Murray (1984) for general monopoles. Because $\operatorname{dim} \pi_{*} E$ is constant it follows from Grauert's theorem (Hartshorne, 1977) that $\pi_{*} E$ is locally free and therefore a vector bundle. This enables us to define a map

by

$$
E^{0} \otimes \operatorname{det} \pi_{*} E \rightarrow E^{\infty} \otimes \operatorname{det} E^{0}
$$

$$
v \otimes \varphi_{1} \wedge \ldots \wedge \varphi_{n} \rightarrow \sum_{i=1, \ldots, n}(-1)^{i+1} \varphi_{i}(\infty) \otimes v \wedge \varphi_{1}(0) \wedge \ldots \widehat{\varphi_{i}(0)} \ldots \wedge \varphi_{n}(0) .
$$

Here $v \in E^{0}(\gamma)$ for some $\gamma \in T P_{1}$ and $\varphi_{1}, \ldots, \varphi_{n}$ is a basis of $H^{0}\left(\pi^{-1}(\gamma), E\right)$.

Notice that the determinant of the evaluation map $\pi_{*} E \rightarrow E^{0}$ defines the curve $S_{0}$ so that because $\operatorname{det} E^{0} \simeq \mathcal{O}$ we have $\operatorname{det} \pi_{*} E \simeq \mathcal{O}\left(-2 k_{0}\right)$.

The remaining spectral curves $S_{1}, \ldots, S_{n-1}$ can be defined as the divisors of the maps

$$
\varphi_{i}: \Lambda^{i} E_{i}^{\infty} \otimes \operatorname{det} \pi_{*} E \rightarrow \Lambda^{i} E^{0} / E_{n-i}^{0} \otimes \operatorname{det} E^{\infty}
$$

defined in a similar manner to (4.5). Hence we have $S_{i}$ defined by $\varphi_{i} \in H^{0}\left(T P_{1}\right.$, $\left.\mathcal{O}\left(2 k_{0}+\ldots+2 k_{i}\right)\right)$. The spectral curve $S_{i}$ is defined to be the fibres where there is a section $\phi$ of $E$ over the fibre with $\phi(\infty) \in E_{i}^{\infty}$ and $\phi(0) \in E_{n-i}^{0}$ for $i=0, \ldots, n-1$. In this manner we could also define $S_{n}$ but it would just be $S_{0}$. This reflects the fact that the Dynkin diagram of the Kac-Moody algebra $\widetilde{\Omega} S U(N)$ is Fig. 1, below.

The analogue of the fundamental weights for this Kac-Moody algebra are $\lambda_{0}=\gamma, \lambda_{1}=\gamma+x_{1}, \ldots, \lambda_{n-1}=\gamma+x_{1}+\ldots+x_{n-1}$. As in Murray (1984) we define the magnetic charges of the monopole to be $m_{0}=k_{0}, m_{1}=k_{0}+k_{1}, \ldots, m_{n-1}=k_{0}+k_{1}$ $+\ldots+k_{n-1}$. The fact that the $S_{i}$ are compact means that $\varphi$ cannot vanish on all of $T P_{1}$, so we must have

$$
m_{i} \geqq 0 \forall j=0,1, \ldots, n-1 .
$$

For $S U(n)$ monopoles the spectral curves have the property that their intersections $S_{i} \cap S_{j}$, when $i$ and $j$ were joined on the Dynkin diagram, have a natural splitting $S_{i}$ $\cap S_{j}=S_{i j} \cup S_{j i}$, and for each $i$ the union $S_{i+1, i} \cup S_{i-1, i}$ is the divisor class of the line bundle

$$
L^{\mu_{i}-\mu_{i-1}}\left(m_{i-1}+m_{i+1}\right)
$$

restricted to the curve $S_{i}$.

Fig. 1

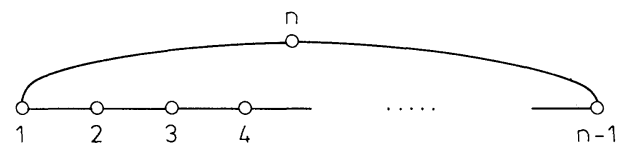


For calorons exactly the same thing occurs except that we have to take into account the Dynkin diagram of the Kac-Moody algebra and we find that we have

$$
S_{0} \cap S_{1}=S_{0,1} \cup S_{1,0} \ldots S_{n-1} \cap S_{0}=S_{n-1,0} \cup S_{0, n-1} .
$$

If $[D]$ denotes the line bundle defined by a divisor $D$ then

$$
\left[S_{1,0} \cup S_{n-1,0}\right]=L^{\mu_{0}-\mu_{1}+\mu_{n}}\left(m_{1}+m_{n-1}\right)
$$

restricted to $S_{0}$,

$$
\left[S_{0,1} \cup S_{2,1}\right]=L^{\mu_{1}-\mu_{2}}\left(m_{0}+m_{2}\right)
$$

restricted to $S_{1}$,

and

$$
\left[S_{n-2, n-1} \cup S_{0, n-1}\right]=L^{\mu_{n-1}-\mu_{n}}\left(m_{n-2}+m_{0}\right)
$$

restricted to $S_{n-1}$.

If $V$ and $W$ are subspaces of $E^{\infty}(\gamma)$ and $E^{0}(\gamma)$, then let $|V \cap W|$ denote the dimension of the space of holomorphic sections $\varphi$ of $E$ on $\pi^{-1}(\gamma)$ with $\varphi(\infty) \in V$ and $\varphi(0) \in W$. Then the sets $S_{i, j}$ are defined as follows

$$
\begin{gathered}
S_{0,1}=\left\{\gamma|| E_{0}^{\infty}(\gamma) \cap E_{n-1}^{0}(\gamma) \mid \geqq 1\right\}, S_{1,0}=\left\{\gamma|| E_{1}^{\infty}(\gamma) \cap E_{n}^{0}(\gamma) \mid \geqq 2\right\}, \\
\vdots \\
S_{p-1, p}=\left\{\gamma|| E_{p-1}^{\infty}(\gamma) \cap E_{n-p}^{0}(\gamma) \mid \geqq 1\right\}, S_{p, p-1}=\left\{\gamma|| E_{p}^{\infty}(\gamma) \cap E_{n-p+1}^{0}(\gamma) \mid \geqq 2\right\}, \\
\vdots \\
S_{n-1,0}=\left\{\gamma|| E_{n-1}^{\infty}(\gamma) \cap E_{0}^{0}(\gamma) \mid \geqq 1\right\}, S_{0, n-1}=\left\{\gamma|| E_{n}^{\infty}(\gamma) \cap E_{1}^{0}(\gamma) \mid \geqq 2\right\} .
\end{gathered}
$$

For the curves $S_{1}, \ldots, S_{n-1}$ the construction of the divisors is done in the same way as in Murray (1984). Consider then the curve $S_{0}$. Over $S_{0}$ there are two line bundles $M_{0}\left(M_{\infty}\right)$, the bundle of all sections of $E$ over a fibre vanishing at $0(\infty)$. Because $\mathscr{T}=L^{\mu_{0}}$, then for each $\gamma \in T P_{1} L^{-\mu_{0}}(\gamma)$ is the space of linear functions from the fibre of $\mathscr{T}$ over $\gamma$ into $C$ which are 0 at 0 and $\infty$ at infinity and hence there is a natural identification

$$
M_{\infty} \otimes L^{-\mu_{0}} \simeq M_{0} .
$$

The set $S_{n-1,0}$ is the divisor of the map $M_{0} \rightarrow E^{\infty} / E_{n-1}^{\infty}=L^{\mu_{n}}\left(k_{n}\right)$, and so we have $\left[S_{n-1,0}\right]=M_{0}^{*} \otimes L^{\mu_{n}}\left(k_{n}\right)$. Similarly for $S_{0,1}$ we deduce that $\mathcal{O}\left(2 m_{0}\right)=\left[S_{1}\right]_{\mid S_{0}}=\left[S_{0,1}\right.$ $\left.\cup S_{1,0}\right]=\left[S_{1,0}\right] M_{\infty}^{*} \otimes L^{\mu_{1}}\left(k_{1}\right)$, and hence that

$$
\left[S_{n-1,0} \cup S_{1,0}\right]=M_{0}^{*} M_{\infty} L^{\mu_{n}-\mu_{1}}\left(-k_{n}+2 k_{0}+k_{1}\right)=L^{\mu_{0}-\mu_{1}+\mu_{n}}\left(m_{1}+m_{n-1}\right) .
$$

If (4.9) and (4.10) are the only constraints on the spectral curves, then using the same calculation as in Murray (1984) the dimension of the moduli space of calorons can be conjectured to be

$$
4 \sum_{i=1}^{n} m_{i}-n+N
$$

where $N$ is the number of vanishing $m_{i}$. 
Notice that if $m_{0}=0$ the bundle $E$ is trivial on the fibres of $\mathscr{T} \rightarrow T P_{1}$ and can be pushed down to $T P_{1}$ and used to construct an $S U(n)$ monopole. The spectral data $\left\{S_{i}, S_{i} \cap S_{i+1} \mid i \in Z / n Z\right\}$ also reduces to the spectral data of an $S U(n)$ monopole.

\section{Kac-Moody Monopoles and the Twistor Correspondence}

We shall use the results of the previous section to show that the twistor correspondence of Murray (1984) applies to Kac-Moody monopoles.

Recall from Murray (1984) that a monopole for a compact group $K$ with reduction at infinity to a maximal torus $T$ is determined by a holomorphic principal bundle $Q$ on $T P_{1}$. This bundle has structure group $G$, the complexification of $K$, and two reductions to Borel sub-bundles. These reductions determine a section of the fibration $Q \times{ }_{G} G / B$ and a cell decomposition of each fibre. The manner in which the section intersects the cells defines the spectral curves $S_{1}, \ldots, S_{n}$ and the decomposition $S_{i} \cap S_{j}=S_{i j} \cup S_{j i}$ when $i$ and $j$ are joined on the Dynkin diagram.

If we regard $\Omega S U(n)$ as an analogue of $K$ we wish to define its complexification. Think of $C^{n} \times C^{\times}$as a trivial $C^{n}$ bundle over $C^{\times}$and consider the group $\mathscr{G}$ of all determinant one holomorphic bundle isomorphisms $C^{n} \times C^{\times} \rightarrow C^{n} \times C^{\times}$whose induced action on $C^{\times} \rightarrow C^{\times}$extends to 0 and $\infty$ and fixes them, so must therefore be multiplication by a non-zero complex number. The group $\mathscr{G}$ is the semidirect product of $\Omega_{C} S L(n, C)$, the group of all holomorphic maps from $C^{\times}$to $S L(n, C)$ and the group $C^{\times}$. If $(f, z),(g, w)$ are two elements of $\mathscr{G}$ the product is

$$
(g, w)(f, z)=(g(z-) f(-), w z) .
$$

Now for any $\gamma \in T P$, define $2(\gamma)$ to be all holomorphic bundle isomorphisms $C^{n}$ $\times C^{\times} \rightarrow E \mid \pi^{-1}(\gamma)$ whose induced action $\pi^{-1}(\gamma) \rightarrow C^{\times}$extends to zero and infinity and stabilizes them. Notice we do not require that the isomorphism extends across zero and infinity. The bundle $\mathscr{Q}$ is then a $\mathscr{G}$ bundle on $T P_{1}$ where $\mathscr{G}$ acts by premultiplication.

If $B$ is the group of upper triangular matrices and $B^{-}$the lower, then the analogue of these Borels for $\mathscr{G}$ are $\mathscr{B}$ the subgroup of maps $C^{\times} \rightarrow S L(n, C)$ extending to $\infty$ and taking values in $B$ there, and $\mathscr{B}^{-}$the subgroup of maps extending to 0 and taking values in $B^{-}$there. The reduction $\mathscr{R}^{+}$is the set of all isomorphisms $C^{n} \times C^{\times} \rightarrow E_{\mid \pi^{-1}(\gamma)}$ which extend to $\infty$ and send the flag in $C^{n}$ stabilized by $B$ to the flag in $E^{\infty}$. A similar definition at 0 defines $\mathscr{R}^{-}$a $\mathscr{B}^{-}$ reduction.

These bundles then provide the analogues of the bundles in the finite dimensional case. Constructing the spectral curves becomes a question of comparing the Bruhat cells in the fibres of $\mathscr{2} \times_{\mathscr{G}} \mathscr{G} / \mathscr{B}$ which have finite dimension with the Birkhoff cells which have finite co-dimension.

At this point we can make clear our assertion that requiring the bundle to split like $\mathcal{O}(1) \oplus \mathcal{O}(-1) \oplus \mathcal{O} \ldots \oplus \mathscr{O}$ is a reasonable assumption. From Pressley and Segal (1987) we see that the cell in $\mathscr{G} / \mathscr{B}$ which contains the bundles splitting like $\mathcal{O}\left(a_{1}\right) \oplus \ldots \oplus \mathcal{O}\left(a_{n}\right)$ for $a_{1} \geqq \ldots \geqq a_{n}$ has complex codimension

$$
\sum_{i<j}\left|a_{i}-a_{j}\right|-n(i, j)
$$


where $n(i, j)$ is zero if $\left|a_{i}-a_{j}\right|=0$ and 1 otherwise. It is not hard to see that the cells containing bundles that do not split like $\mathcal{O} \oplus \ldots \oplus \mathcal{O}$ or $\mathcal{O}(1) \oplus \mathcal{O} \ldots \mathcal{O} \oplus \mathcal{O}(-1)$ have codimension $>2$, so a generic section of $2 \times{ }_{g} \mathscr{G} / \mathscr{B}$ shouldn't intersect them. Of course the sections we are considering are very special and may intersect these cells, but at least this makes the assumption reasonable.

In constructing the spectral curves for general monopoles it was very convenient to define them by looking at highest weight modules. To do this for $\mathscr{G}$ we need to lift the bundle to the central extension $\hat{\mathscr{G}}$ of the loop group. This should be possible for the following reason. We have

$$
0 \rightarrow C^{\times} \rightarrow \hat{\mathscr{G}} \rightarrow \mathscr{G} \rightarrow 0
$$

and a bundle 2 trivial on real sections. We would like to lift to a bundle $\widehat{\mathscr{Q}}$ trivial on real sections. Because (5.3) is a central extension the obstruction to lifting is in $H^{2}\left(T P_{1}, \mathcal{O}^{\times}\right)=0$, so a lift can be defined. If we restrict to $P^{1}$ a real section the obstruction to triviality will be the chern class in $H^{1}\left(P^{1}, \mathcal{O}^{\times}\right)$and this is independent of the real section. If we twist $\widehat{\mathscr{Q}}$ by the correct line bundle we can remove this obstruction.

Proving that in $R^{3}$ a solution of the Bogomolny equations lifts to $\widetilde{\Omega} S U(n)$ means finding $(a, \phi)$ a one form and function satisfying

$$
d a+\operatorname{tr} \int \frac{\partial A}{\partial \theta} \wedge A d \theta=* d \phi+\operatorname{tr} \int \frac{\partial * A}{\partial \theta} \Phi d \theta .
$$

The argument above indicates that such an $(a, \theta)$ should exist but doesn't serve as a proof as we do not have a rigorous proof of the twistor correspondence for $\widehat{\mathscr{G}}$ bundles.

\section{Constructing the Caloron from the Spectral Data}

In this section we indicate how the method used in Hurtubise and Murray (1988a) can be adapted to the case of calorons to show that they are determined by their spectral data.

We define an infinite dimensional bundle $\mathscr{F}$ over minitwistor space which is the push down of the bundle $E$ restricted to $\hat{\mathscr{T}}$, except that we allow only those sections along the fibre that have finite poles or zeros at $\mathscr{T}^{0}$ and $\mathscr{T}^{\infty}$. The fibre of this bundle then looks like $C\left[z, z^{-1}\right] \otimes C^{n}$.

This bundle has two natural filtrations. Let $z$ be a linear co-ordinate along some fibre which approaches zero at zero and infinity at infinity. We define $\mathscr{F}_{p, q}^{\infty}$ to be the sub-bundle of sections $\varphi$ of $E$ along the fibre for which $\left(1 / z^{p}\right) \varphi$ is finite at infinity and takes its value in $E_{q}^{\infty}$. Similarly define $\mathscr{F}_{-p, n-1}^{0}$ to be the sub-bundle of sections $\varphi$ of $F$ along the fibre for which $\left(1 / z^{p}\right) \varphi$ is finite at zero and takes its value in $E_{n-q}^{0}$. Less precisely

$$
\mathscr{F}_{p, q}^{0}=\ldots z^{p-1} E^{\infty}+z^{p} E_{q}^{\infty}
$$

and

$$
\mathscr{F}_{-p, n-q}^{0}=z^{p} E_{n-q}^{0}+z^{p+1} E^{0}+\ldots
$$


From the definition of the spectral curves we see that $\mathscr{F}_{p, q}^{\infty}$ and $\mathscr{F F}_{-p, n-p}^{0}$ have non-zero intersection only on the $p^{\text {th }}$ spectral curve. We shall restrict ourselves to the case of general calorons, that is, those whose spectral curves intersect finitely. This implies the genericity assumption we have already made on the splitting type of the bundle $E$. With this assumption these two sub-bundles intersect in a one dimensional space over the spectral curve $S_{p}$.

We want to define now the sequence used in Hurtubise and Murray 1988a to recover a monopole from its spectral data. Throughout we shall be using the same symbol for a vector bundle and its sheaf of sections and stress that what follows is a sequence of sheaves.

$$
\begin{aligned}
& \oplus \quad \frac{\mathscr{F}}{\mathscr{F}_{p, q}^{\infty}+\mathscr{F}_{-p, n-q}^{0}} \\
& 0 \rightarrow \mathscr{F} \rightarrow \frac{\mathscr{F}}{\mathscr{F}_{p, q}^{\infty}+\mathscr{F}_{-p, n-q-1}^{0}} \quad \rightarrow \quad \rightarrow \quad \rightarrow 0 \\
& \oplus \\
& \frac{\mathscr{F}}{\mathscr{F}_{p, q+1}^{\infty}+\mathscr{F}_{-p, n-q-1}^{0}}
\end{aligned}
$$

The first map here is just a collection of projections into the various pieces of the direct sum. We note that because the sections in $\mathscr{F}$ have only finite poles and zeroes at zero and infinity only a finite number of these projections are non-zero for any particular element. The second map is more complicated and is obtained by using the two projections

$$
\begin{gathered}
\frac{\mathscr{F}}{\mathscr{F}_{p, q}^{\infty}+\mathscr{F}_{-p, n-q-1}} \rightarrow \frac{\mathscr{F}}{\mathscr{F}_{p, q}^{\infty}+\mathscr{F}_{-p, n-q}^{0}}, \\
\frac{\mathscr{F}}{\mathscr{F}_{p, q}^{\infty}+\mathscr{F}_{-p, n-q-1}^{0}} \rightarrow \frac{\mathscr{F}_{\mathscr{F}_{p, q+1}^{\infty}+\mathscr{F}_{-p, n-q-1}^{0}}^{0}}{\mathscr{F}_{-p}},
\end{gathered}
$$

with alternating signs so that the composition of the two maps in (6.3) is zero.

Because the spectral curves are never the whole of minitwistor space the first map is an injection of sheaves. To prove that this sequence is exact then we need only to prove that something killed by the second map comes from an element of $\mathscr{F}$. Consider then an element $y=\left\{y_{p, q}+\mathscr{F}_{p, q}^{\infty}+\mathscr{F}_{-p, n-q-1}^{0}\right\}$ which is killed by the second map.

This means that there exist elements $x_{p, q}^{\infty} \in \mathscr{F}_{p, q}^{\infty}$ and $x_{p, q}^{0} \in \mathscr{F}_{p, q}^{0}$ which satisfy

$$
y_{p, q+1}+y_{p, q}=x_{p, q}^{\infty}-x_{p, n-q-1}^{0},
$$

where the labels identify where the $x$ 's live. If we define new $\hat{y}$ 's by

$$
\hat{y}_{p, q}=y_{p, q}+x_{p, q}^{\infty}+x_{p, q-1}^{\infty}+\ldots+x_{-p, n-q-1}^{0}+x_{-p, n-q-2}^{0}+\ldots,
$$

then these are in the same cosets as the $y_{p, q}$ because of the way the filtrations are nested. Moreover because the elements of the direct sum are only finitely supported, this sum is finite and so these $\hat{y}$ 's are well defined and a straightforward calculation shows that

$$
\hat{y}_{p, q}=\hat{y}_{p, q+1},
$$

so that they define a unique $\hat{y}$ whose image is the element $y$. 
To show that the caloron is determined by its spectral data we only need show that the spectral data determines the last two columns of the exact sequence and the map between them. The caloron is then recovered as the kernel of this sequence. First let us identify the quotients of the filtration by observing that because $\hat{\mathscr{T}}$ is the line bundle $L^{\mu_{0}}$ with the zero section removed, the linear coordinates $z$ used above are elements of the dual bundle $L^{-\mu_{0}}$ and we can define an isomorphism

$$
\begin{aligned}
\mathscr{F}_{p, q}^{\infty} / \mathscr{F}_{p, q-1}^{\infty} & \simeq L^{-p \mu_{0}} \otimes E_{q}^{\infty} / E_{q-1}^{\infty}=L^{-p \mu_{0}+\mu_{q}}\left(-k_{q}\right), \\
\varphi & \mapsto\left(z^{p}\right) \otimes\left(z^{-p} \varphi\right) .
\end{aligned}
$$

Similarly

$$
\mathscr{F}_{p, q}^{0} / \mathscr{F}_{p, q-1}^{0} \simeq L^{p \mu_{0}+\mu_{n-q+1}}\left(-k_{n-q+1}\right) .
$$

Notice also that the map

$$
\begin{aligned}
& \mathscr{F} \rightarrow L^{-r \mu_{0}} \mathscr{F}, \\
& \varphi \mapsto z^{r} \otimes\left(z^{r} \varphi\right)
\end{aligned}
$$

sends $\mathscr{F}_{p, q}^{\infty}$ to $\mathscr{F}_{p+r, q}^{\infty}$ and $\mathscr{F}_{p, q}^{0}$ to $\mathscr{F}_{p+r, q}^{0}$. So it suffices to identify the pieces of the sequence and the maps when $p=0$.

The sheaf

$$
\frac{\mathscr{F}}{\mathscr{F}_{0, q}^{\infty}+\mathscr{F}_{0, n-q}^{0}}
$$

is, by our genericity assumptions, a line bundle on the curve $S_{q}$, and the natural projection of $\mathscr{F}_{0, q+1}^{\infty}$ onto this line bundle factors to give a map

$$
\mathscr{F}_{0, q+1}^{\infty} \rightarrow \frac{\mathscr{F}}{\mathscr{F}_{0, q}^{\infty}+\mathscr{\mathscr { F }}{ }_{0, n-q}^{0}}
$$

which vanishes precisely at the points $S_{q+1, q}$ from (4.11). Hence we have

$$
\frac{\mathscr{F}}{\mathscr{F}_{0, q}^{\infty}+\mathscr{F}_{0, n-q}^{0}} \simeq L^{\mu_{q+1}}\left(-k_{q}\right)\left[-S_{q+1, q}\right] \simeq L^{\mu_{q}}\left(m_{q-1}+m_{q}\right)\left[-S_{q-1, q}\right]
$$

using (4.10).

To identify the middle terms consider the map

$$
\pi_{*} E \rightarrow \frac{\mathscr{F}_{0, n}^{\infty}}{\mathscr{F}_{0, q}^{\infty}} \oplus \frac{\mathscr{F}_{0, n}^{0}}{\mathscr{F}_{0, n-q-1}^{0}}, \quad a \mapsto(a,-a) .
$$

Because of the genericity assumption $\pi_{*} E$ is a vector bundle and we can evaluate at infinity and zero to identify this map as

$$
\pi_{*} E \rightarrow \frac{E^{\infty}}{E_{q}^{\infty}} \oplus \frac{E^{0}}{E_{n-q-1}^{0}},
$$

a map of vector bundles. This map of vector bundles is injective as a map of sheaves, in fact the image of the fibre $\left(\pi_{*} E\right)(\gamma)$ is always $n$ dimensional except if $\gamma \in S_{q, q+1}$. Again, because of the genericity assumption on the caloron $\mathscr{F}=\mathscr{F}_{0, n}^{\infty}$ $+\mathscr{F}_{0, n}^{0}$, and the sheaf quotient of this map is

$$
\mathscr{Q}=\frac{\mathscr{F}}{\mathscr{\mathscr { F }}_{0, q}^{0}+\mathscr{F}_{0, n-q-1}^{0}} .
$$


It follows that we have a map of sheaves

$$
\mathscr{Q} \rightarrow\left(\operatorname{det} \pi_{*} E\right)^{*} \otimes \Lambda^{n-q} \frac{E^{\infty}}{E_{q}^{\infty}} \otimes \Lambda^{q+1} \frac{E^{0}}{E_{n-q-1}^{0}},
$$

and because (6.13) is an injective bundle map away from $S_{q, q+1}, \mathscr{Q}$ is locally free away from $S_{q, q+1}$ and (6.15) is an isomorphism away from $S_{q, q+1}$. The map in (6.15) is therefore an injection of sheaves. We want to show that

$$
\begin{aligned}
0 \rightarrow \mathscr{Q} & \rightarrow\left(\operatorname{det} \pi_{*} E\right)^{*} \otimes \Lambda^{n-q} \frac{E^{\infty}}{E_{q}^{\infty} \otimes \Lambda^{q-1} \frac{E^{0}}{E_{n-q-1}^{0}}} \\
& \left.\rightarrow\left(\operatorname{det} \pi_{*} E\right)^{*} \otimes \Lambda^{n-q} \frac{E^{\infty}}{E_{q}^{\infty}} \otimes \Lambda^{q-1} \frac{E^{0}}{E_{n-q-1}^{0}}\right|_{S_{q, q+1}} \rightarrow 0
\end{aligned}
$$

is an exact sequence of sheaves so that

$$
\mathscr{Q} \simeq L^{\mu_{q+1}}\left(m_{q}+m_{q+1}\right) \otimes \mathscr{I}\left(-S_{q, q+1}\right),
$$

where $\mathscr{I}\left(-S_{q, q+1}\right)$ is the ideal sheaf of functions vanishing on $S_{q, q+1}$.

We have seen that the sequence is exact off $S_{q, q+1}$ and that the first and second maps are injective and surjective. Moreover it is straightforward to check that the image of 2 restricted to $S_{q, q+1}$ vanishes. If $x \in S_{q, q+1}$ then in a neighbourhood of $x$ we can choose a local section of $\mathscr{F}_{0, q+1}^{\infty}$ not in $\mathscr{F}_{0, q}^{\infty}$, and the image of the element of $\mathscr{Q}$ this produces is a local section vanishing on $S_{q+1}$. Similarly using $\mathscr{F}_{0, n-q}^{0}$ we obtain a local section in the image of $\mathscr{Q}$ vanishing on $S_{q}$. Hence the image of $\mathscr{2}$ contains elements vanishing on $S_{q}$ and $S_{q+1}$, but these intersect in $x$ so the image contains the whole ideal sheaf of $x$, and it follows that the sequence (6.16) is exact.

We have now identified all the spaces in the summands in (6.3) in terms of known line bundles and the spectral data. But what of the maps in $(6.4,6.5)$ ? Under these identifications they become

$$
\begin{gathered}
\left.L^{\mu_{q+1}}\left(m_{q}+m_{q+1}\right) \otimes \mathscr{I}\left(-S_{q, q+1}\right) \rightarrow L^{\mu_{q}}\left(m_{q-1}+m_{q}\right)\right|_{s_{q}}\left[-S_{q-1, q}\right], \\
\left.L^{\mu_{q+1}}\left(m_{q}+m_{q+1}\right) \otimes \mathscr{I}\left(-S_{q, q+1}\right) \rightarrow L^{\mu_{q+1}}\left(m_{q}+m_{q+1}\right)\right|_{s_{q+1}}\left[-S_{q, q+1}\right] .
\end{gathered}
$$

The second map (6.19) is just restriction and the first (6.18) is restriction and multiplication by a meromorphic section of $L^{\mu_{q}-\mu_{q+1}}\left(m_{q-1}-m_{q+1}\right)$ over $S_{q}$ with divisor $S_{q, q+1}-S_{q-1, q}$. This meromorphic section is unique up to scalars from (4.10). We have shown now that the summands and the maps between them are determined by the spectral data (the scalar changes don't matter) and hence we can recover the bundle $\mathscr{F}$ as the kernel of this map. The general caloron is therefore determined by its spectral data.

The proof in Hurtubise and Murray (1988b) which uses the cell structure of the flag manifold could also be used to show that the spectral data determines the caloron. However the structures we have elaborated here are more useful for solving Nahm's equations, see Hurtubise and Murray (1988a) and Garland, Hurtubise, and Murray (to appear).

\section{Kac-Moody Monopoles for Other Loop Groups and Twisted Loop Groups}

The instanton methods by which a bundle is constructed over $\mathscr{T}$ extend to the case where $S U(n)$ is replaced by any simple, compact Lie group $K$. In this case a 
holomorphic principal bundle with structure group $G$, the complexification of $K$, is constructed. By considering the adjoint bundle we can show as in Sect. 3 that this holomorphic bundle $Q$ extends to $\mathscr{T}$ and has a Borel reductions over $\mathscr{T}^{\infty}$ and $\mathscr{T}^{0}$.

The various spectral curves $S_{1}, \ldots, S_{n}$ can now be constructed by looking at the fundamental representations of $G$ as in Murray (1984). However there are some difficulties with the jumping curve $S_{0}$.

The curve $S_{0}$ will be the set of fibres over which $Q$ is not trivial. To see its structure as an algebraic variety it seems necessary to consider the loop group as an algebraic variety and work essentially with infinite dimensional bundles over $T P_{1}$. If instead we choose a representation of $G$ we get an associated bundle $E$ and it has a jumping curve of degree $c_{2}(E)$ which may be greater than the number of points at which $Q$ jumps over a generic $P_{1}$. This would mean we were considering a multiple of $S_{0}$.

For groups other than $S U(n)$ then, it is better to treat the caloron as a KacMoody monopole and use the methods of Murray (1984) and Hurtubise and Murray (1988b) to define the spectral data and show that it determines the general caloron. This can be done, but we have not bothered presenting the details here because of the lack of an existence proof for calorons. The $S U(n)$ case and the methods we have adopted have the advantage that they should allow a proof of existence by relating calorons to rational maps of $P_{1}$ into the flag manifold of the loop group as in Hurtubise and Murray (1988a) and Hurtubise (1988).

The theory of Kac-Moody monopoles extends also to the twisted Kac-Moody algebras. Certain Lie algebras $L K$ admit automorphisms $\chi$ such that $\chi^{d}=1$, where $d=2$ or 3 . They can be determined by seeing which Dynkin diagrams admit such automorphisms. We can then consider equivariant maps $f: S^{1} \rightarrow L K$ such that $f\left(e^{i \theta}, e^{i 2 \pi / d}\right)=\chi\left(f\left(e^{i \theta}\right)\right)$, and these with the appropriate extension form the KacMoody algebra. A twisted Kac-Moody monopole is therefore a periodic instanton invariant under this additional symmetry. The automorphism therefore acts on the holomorphic bundle and the spectral curves should become identified in exactly the same way that the Dynkin diagram of $L K$ becomes identified to form the Dynkin diagram of the twisted Kac-Moody monopole.

\section{Nahm's ADHM Construction for Kac-Moody Monopoles}

We give a brief discussion of how Nahm's ADHM construction applies to calorons to strengthen the analogy with the case of finite dimensional monopoles.

Starting with a periodic instanton Nahm (1983) considers the Dirac equations

$$
\begin{aligned}
& D_{z}=D_{A}-\left(\Phi+\mu_{0} \frac{\partial}{\partial \theta}+i z\right), \\
& D_{z}^{+}=D_{A}+\left(\Phi+\mu_{0} \frac{\partial}{\partial \theta}+i z\right),
\end{aligned}
$$

where $D_{A}$ is the covariant Dirac operator.

Let $\tilde{W}_{z}$ be the $L^{2}$ kernel of $D_{z}^{+}$. Then observe that multiplication by $e^{-i n}, n \in Z$ defines a map

$$
\tilde{W}_{z} \rightarrow \tilde{W}_{z+\mu_{0} n} .
$$


Taking the quotient defines a "bundle" on $R / \mu_{0} Z \simeq S^{1}$. We use quotes because the rank of the bundle may jump at the points

for $j=1, \ldots, m$.

$$
\mu_{j} \bmod \mu_{0} Z
$$

The connection $\nabla_{\theta}$ and Higgs fields $T_{i}, i=1,2,3$ can be defined as for the finite dimensional monopoles as

and

$$
\tilde{\nabla}_{z}=\pi \circ \frac{\partial}{\partial \theta}
$$

$$
\widetilde{T}_{i}=\pi \circ x_{i}
$$

where $\pi$ is the orthogonal projection from $L^{2}\left(R^{3} \times S^{1}, C^{n} \otimes C^{2}\right) \rightarrow \tilde{W}$. These are covariant under the action of $\mu_{0} Z$ as above and descend to a connection and Higgs fields on $W \rightarrow S^{1}$ which satisfy Nahm's equations.

The inverse construction proceeds in a similar fashion.

Notice that this construction agrees with the conjecture of Atiyah that we should view Nahm's equations as being solved on the dual of the Dynkin diagram.

Acknowledgements. Both authors would like to thank the Mathematical Sciences Research Institute for its hospitality and the National Science Foundation for its support. The second author also acknowledges the support of the National Research Fellowships scheme.

\section{References}

Atiyah, M.F., Hitchin, N.J., Singer, I.M.: Self-duality in four-dimensional Riemannian geometry. Proc. R. Soc. Lond. A 362, 425-461 (1978)

Coppel, W.A.: Stability and asymptotic behaviour of differential equations. Boston, DC: Heath 1965

Chakrabarti, A.: Periodic generalizations of static self-dual SU(2) gauge fields. Phys. Rev. D 35, 2, 696-706 (1987)

Garland, H., Hurtubise, J.C., Murray, M.K.: Calorons and rational maps. (to appear)

Hartshorne, R.: Algebraic geometry. Berlin, Heidelberg, New York: Springer 1977

Hitchin, N.J.: Monopoles and geodesics. Commun. Math. Phys. 83, 579-602 (1982)

Hitchin, N.J.: On the construction of monopoles. Commun. Math. Phys. 89, 145-190 (1983)

Hitchin, N.J., Murray, M.K.: Spectral curves and the ADHM construction. Commun. Math. Phys. 114, 463-474 (1988)

Hurtubise, J.C.: Monopoles and rational maps. (to appear in Commun. Math. Phys. 1989)

Hurtubise, J.C., Murray, M.K.: On the construction of classical monopoles. (to appear in Commun. Math. Phys. 1988a)

Hurtubise, J.C., Murray, M.K.: Monopoles and their spectral data (to appear 1988b)

Macdonald, I.G.: Affine Lie algebras and modular forms. Seminaire Bourbaki 33e annee 1980/81 no 577

Murray, M.K.: Non-abelian magnetic monopoles. Commun. Math. Phys. 96, 539-565 (1984)

Nahm, W.: Self-dual monopoles and calorons. Preprint Bonn-University Bonn-HE-83-16. September 1983

Segal, G., Pressley, A.N.: Loop groups. Oxford: Oxford University Press 1987

Communicated by A. Jaffe 
